QUARTERLY OF APPLIED MATHEMATICS

VOLUME LXVIII, NUMBER 3

SEPTEMBER 2010, PAGES 439-458

S 0033-569X(2010)01212-7

Article electronically published on May 19, 2010

\title{
VARIATIONAL PROBLEMS IN WEIGHTED SOBOLEV SPACES ON NON-SMOOTH DOMAINS
}

\author{
BY \\ ANA MARIA SOANE (Department of Mathematics and Statistics, University of Maryland, \\ Baltimore County, 1000 Hilltop Circle, Baltimore 21250, Maryland) \\ AND \\ ROUBEN ROSTAMIAN (Department of Mathematics and Statistics, University of Maryland, \\ Baltimore County, 1000 Hilltop Circle, Baltimore 21250, Maryland)
}

Abstract. We study the Poisson problem $-\Delta u=f$ and the Helmholtz problem $-\Delta u+\lambda u=f$ in bounded domains with angular corners in the plane and $u=0$ on the boundary. On non-convex domains of this type, the solutions are in the Sobolev space $H^{1}$ but not in $H^{2}$ in general, even though $f$ may be very regular. We formulate these as variational problems in weighted Sobolev spaces and prove existence and uniqueness of solutions in what would be weighted counterparts of $H^{2} \cap H_{0}^{1}$.

The specific forms of our variational formulations are motivated by, and are particularly suited to, applying a finite element scheme for solving the time-dependent NavierStokes equations of fluid mechanics.

1. Introduction. Consider the Poisson problem in an open and bounded domain $\Omega$ in $\mathbb{R}^{n}$ :

$$
\begin{aligned}
-\Delta u=f & \text { in } \Omega, \\
u=g & \text { on } \partial \Omega .
\end{aligned}
$$

The operator $A: u \mapsto(f, g)$ is an isomorphism from $H^{s}(\Omega)$ to $H^{s-2}(\Omega) \times H^{s-1 / 2}(\partial \Omega)$ for $s \geq 2$, provided that the boundary is of class $C^{s}$. See, e.g., Lions and Magenes [18, Theorem 5.5.4], Gilbarg and Trudinger [8, Theorem 8.13], Evans [7, Theorem 6.3.5]. Much work has been done on extending this to cases where the regularity of the boundary is less than $C^{s}$. A wealth of information about this and further references to the literature

Received August 12, 2008.

2000 Mathematics Subject Classification. Primary 35J20; Secondary 35Q30, 46E35, 76D05, 65N30.

Key words and phrases. Poisson problem, Helmholtz problem, corner singularities, weighted Sobolev spaces, finite elements, Navier-Stokes equations.

Current address: MOX, Dipartimento di Matematica, Politecnico di Milano, Piazza L. Da Vinci 32, 20133 Milano, Italy.

E-mail address: asoane@umbc.edu

E-mail address: rostamian@umbc.edu 
are available in Kondrat'ev [12, Kondrat'ev and Oleŭnik 13, Grisvard 9, 10, Dauge 4, Kufner [16, Kufner and Sändig [17, and Kozlov, Maz'ya, and Roßmann [14, 15. For results specific to polyhedral domains, see von Petersdorff and Stephan 25], Nicaise [20, and the survey article by Dauge 6 .

In this work we present variational formulations for the Poisson problem $(-\Delta u=f)$ and the Helmholtz problem $(-\Delta u+\lambda u=f)$ on non-smooth domains in $\mathbb{R}^{2}$ by applying the machinery for weighted Sobolev spaces developed in [12, 14, 17. The novel aspect of this study is the non-traditional variational formulation of two elliptic problems (see (3.2), (3.3), (4.2), and (4.4)) which are motivated by a desire to extend the numerical scheme for solving the Navier-Stokes equations developed by Liu, Liu, and Pego [19 to non-smooth domains. Their algorithm, which calls for solving a Poisson problem in $H^{1}$ and a Helmholtz problem in $H^{2}$ at each discretized time step, converges and is unconditionally stable on domains with sufficiently smooth boundaries. A naive application of the algorithm in non-convex polygonal domains (flow over a step, for example) produces incorrect solutions because the velocity is not in $H^{2}$ near reentrant corners. We propose a modified version of the algorithm that applies appropriate weights near reentrant corners to compensate for singularities. Our analysis of the Poisson and Helmholtz problems leads to a version of the algorithm in [19] in weighted Sobolev spaces that is suitable for solving the Navier-Stokes equations in polygonal domains with reentrant corners.

The plan of this article is as follows. In Section 2 we summarize definitions and theorems pertaining to weighted Sobolev spaces, mostly from [14], that will be needed in the sequel. In Section 3 we introduce our formulation of the Poisson problem (see (3.2) and (3.3)) that extends the traditional $H^{2}$ formulation to domains with corners. We show the existence and uniqueness of solutions and establish their relationship to the usual $H^{1}$ formulation. We repeat this for equations of Helmholtz type in Section 4 (see (4.2) and (4.4)). In Section [5 we apply the variational formulations developed in the previous sections to adapt the iterative algorithm of Liu, Liu, and Pego [19] to solving the time-dependent Navier-Stokes equations to non-convex polygonal domains. The well-posedness of the modified algorithm follows from our analysis of the Poisson and Helmholtz problems in Sections 3 and 4 . We do not address the issue of convergence of the iterates in this article. We do, however, provide numerical evidence in Section 6 that the iteration does produce the expected solution. In addition to reporting results for the Navier-Stokes equations, Section [6] contains convergence studies of two benchmark problems for the Poisson and Helmholtz equations on L-shaped domains, where numerical solutions are compared to a priori known exact solutions. These serve to validate our weighted Sobolev space finite element solver which is described elsewhere 22. For the Poisson and Helmholtz problems, the convergence of our finite element method is shown in 223. and error estimates and optimal rates of convergence are established in [24].

To put this work in context, let us note that there are at least two distinct approaches to dealing with corner singularities. In one approach, as expounded in Grisvard [10], the solution is decomposed into the sum of a "regular part" which is in $H^{2}$ or better, and a "singular part" which is in $H^{1}$. Aziz and Kellogg [1] follow a similar method to construct distributional solutions in $L_{2}$ corresponding to rough data. In the other approach, as exemplified by [14, the solution is considered as a whole, as a member of 
an extended function space that accommodates the singularities and enables the use of variational formulations in the familiar functional analytic settings. The work presented here follows the latter approach.

2. Preliminaries. The weighted Sobolev spaces in a plane wedge are defined in Section 2.1. These are extended to general bounded domains with angular points in Section 2.2. In Section 2.3 we recall a regularity result for the Poisson problem in domains with angular points [14 which will be used extensively in the proofs of our analytical results.

2.1. Weighted Sobolev spaces in a wedge. Let $\mathcal{K}=\left\{\left(x_{1}, x_{2}\right) \in \mathbb{R}^{2}: r>0,0<\theta<\omega\right\}$ be a plane wedge centered at the origin. Here $r, \theta$ are the polar coordinates of the point $\left(x_{1}, x_{2}\right)$ and $\omega \in(0,2 \pi)$. For integer $l \geq 0$ and real $\beta$, the space $V_{2, \beta}^{l}(\mathcal{K})$ is defined as the closure of $C_{0}^{\infty}(\overline{\mathcal{K}} \backslash\{0\})$ with respect to the norm

$$
\|u\|_{V_{2, \beta}^{l}(\mathcal{K})}=\left(\int_{\mathcal{K}} \sum_{|\alpha| \leq l} r^{2(\beta-l+|\alpha|)}\left|D_{x}^{\alpha} u\right|^{2} d x\right)^{1 / 2}
$$

where $D_{x}^{\alpha}=\partial^{|\alpha|} / \partial x_{1}^{\alpha_{1}} \partial x_{2}^{\alpha_{2}},|\alpha|=\alpha_{1}+\alpha_{2}$.

If $l \geq 1, V_{2, \beta}^{l-1 / 2}(\partial \mathcal{K})$ is the space of traces of functions from $V_{2, \beta}^{l}(\mathcal{K})$ on the boundary $\partial \mathcal{K}$ equipped with the norm

$$
\|u\|_{V_{2, \beta}^{l-1 / 2}(\partial \mathcal{K})}=\inf _{v \in V_{2, \beta}^{l}(\mathcal{K})}\left\{\|v\|_{V_{2, \beta}^{l}(\mathcal{K})}:\left.v\right|_{\partial \mathcal{K}}=u\right\} .
$$

We call $\mathcal{K}$, or any translation and rotation of it, a plane wedge of angle $\omega$ and define the spaces $V_{2, \beta}^{l}(\mathcal{K}), V_{2, \beta}^{l-1 / 2}(\partial \mathcal{K})$ accordingly.

2.2. Weighted Sobolev spaces in bounded domains with angular points. Let $\mathcal{G} \subset \mathbb{R}^{2}$ be a bounded domain. Suppose that there exists a finite set $\mathcal{S}=\left\{x^{1}, \ldots, x^{d}\right\}$ of points on the boundary $\partial \mathcal{G}$ such that $\partial \mathcal{G} \backslash \mathcal{S}$ is smooth. Also, assume that for each of the points $x^{j}, j=1, \ldots d$, there exists a neighborhood $\mathcal{U}_{j}$ such that $\mathcal{G} \cap \mathcal{U}_{j}=\mathcal{K}_{j} \cap \mathcal{U}_{j}$, where $\mathcal{K}_{j}$ is a wedge with vertex $x^{j}$. Such a domain is called a domain with angular points.

Let $\zeta_{j}, j=1, \ldots, d$, be infinitely differentiable functions in $\overline{\mathcal{G}}, 0 \leq \zeta_{j}(x) \leq 1$, equal to one in a neighborhood of $x^{j}$ and to zero in $\overline{\mathcal{G}} \backslash \mathcal{U}_{j}$, and set $\zeta_{0}=1-\zeta_{1}-\cdots-\zeta_{d}$. The neighborhoods $\mathcal{U}_{j}, j=1, \ldots, d$, are chosen sufficiently small to be disjoint. The space $V_{2, \vec{\beta}}^{l}(\mathcal{G})$, where $\vec{\beta}=\left(\beta_{1}, \ldots, \beta_{d}\right) \in \mathbb{R}^{d}$ and $l$ is a nonnegative integer, is defined as the set of all functions on $\mathcal{G}$ such that $\zeta_{0} u \in H^{l}(\mathcal{G})$ and $\zeta_{j} u \in V_{2, \beta_{j}}^{l}\left(\mathcal{K}_{j}\right), j=1, \ldots, d$. Here and elsewhere $H^{l}(\mathcal{G})$ is the usual Sobolev space of functions on $\mathcal{G}$ whose weak derivatives of up to order $l$ are square integrable. Equipped with the norm 1

$$
\|u\|_{V_{2, \vec{\beta}}^{l}(\mathcal{G})}=\left(\left\|\zeta_{0} u\right\|_{H^{l}(\mathcal{G})}^{2}+\sum_{j=1}^{d}\left\|\zeta_{j} u\right\|_{V_{2, \beta_{j}}^{l}\left(\mathcal{K}_{j}\right)}^{2}\right)^{1 / 2}
$$

\footnotetext{
${ }^{1}$ This norm is equivalent to the norm $\|u\|_{V_{2, \vec{\beta}}^{l}(\mathcal{G})}=\left\|\zeta_{0} u\right\|_{H^{l}(\mathcal{G})}+\sum_{j=1}^{d}\left\|\zeta_{j} u\right\|_{V_{2, \beta_{j}}^{l}\left(\mathcal{K}_{j}\right)}$ used in [14].
} 
the space $V_{2, \vec{\beta}}^{l}(\mathcal{G})$ is complete. We use the notation $L_{2, \vec{\beta}}(\mathcal{G})$ for the space $V_{2, \vec{\beta}}^{0}(\mathcal{G})$ and define the $L_{2, \vec{\beta}}(\mathcal{G})$ inner product as

$$
(u, v)_{L_{2, \vec{\beta}}(\mathcal{G})}=\int_{\mathcal{G}} \zeta_{0}^{2} u v d x+\sum_{j=1}^{d} \int_{\mathcal{K}_{j}} r_{j}^{2 \beta_{j}} \zeta_{j}^{2} u v d x
$$

where $r_{j}$ is the distance from vertex $x^{j}$.

For $l \geq 1$, the space $V_{2, \vec{\beta}}^{l-1 / 2}(\partial \mathcal{G})$ is defined as the space of traces of functions from $V_{2, \vec{\beta}}^{l}(\mathcal{G})$ on $\partial \mathcal{G} \backslash \mathcal{S}$. The norm in $V_{2, \vec{\beta}}^{l-1 / 2}(\partial \mathcal{G})$ is

$$
\|u\|_{V_{2, \vec{\beta}}^{l-1 / 2}(\partial \mathcal{G})}=\inf _{v \in V_{2, \vec{\beta}}^{l}(\mathcal{G})}\left\{\|v\|_{V_{2, \vec{\beta}}^{l}(\mathcal{G})}:\left.v\right|_{\partial \mathcal{G} \backslash \mathcal{S}}=u\right\} .
$$

We denote by $\stackrel{\circ}{V}_{2, \vec{\beta}}^{l}(\mathcal{G})$ the subspace of zero trace functions from $V_{2, \vec{\beta}}^{l}(\mathcal{G})$,

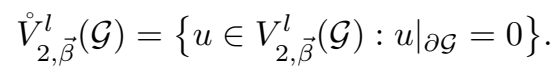

The following imbedding property will be needed in the sequel.

Lemma 2.1 (14, Lemma 6.2.1]). Let $\vec{\beta}=\left(\beta_{1}, \ldots, \beta_{d}\right), \vec{\gamma}=\left(\gamma_{1}, \ldots, \gamma_{d}\right)$ be real $d$-tuples. If $l_{2} \geq l_{1} \geq 0$ and $\beta_{j}-l_{2} \leq \gamma_{j}-l_{1}$ for $j=1, \ldots, d$, then the space $V_{2, \vec{\beta}}^{l_{2}}(\mathcal{G})$ is continuously imbedded into $V_{2, \vec{\gamma}}^{l_{1}}(\mathcal{G})$. If, moreover, $l_{2}>l_{1} \geq 0, \beta_{j}-l_{2}<\gamma_{j}-l_{1}$ for $j=1, \ldots, d$, then this imbedding is compact.

2.3. The Poisson problem in a plane domain with angular points. Let $\mathcal{G} \subset \mathbb{R}^{2}$ be a bounded domain with angular points $x^{1}, \ldots, x^{d}$ with interior angles $\alpha_{j} \in(0,2 \pi)$, $j=1, \ldots, d$. Consider the problem

$$
\begin{aligned}
& -\Delta u=f \quad \text { in } \mathcal{G}, \\
& u=g \text { on } \partial \mathcal{G} \text {, }
\end{aligned}
$$

and denote the operator of this problem by $\mathcal{A}$.

Theorem 2.2 ([14, Theorem 6.6.1]). The operator

$$
\mathcal{A}: V_{2, \vec{\beta}}^{l}(\mathcal{G}) \rightarrow V_{2, \vec{\beta}}^{l-2}(\mathcal{G}) \times V_{2, \vec{\beta}}^{l-1 / 2}(\partial \mathcal{G}),
$$

where $l \geq 2$ and $\vec{\beta}=\left(\beta_{1}, \ldots, \beta_{d}\right) \in \mathbb{R}^{d}$, is an isomorphism if and only if $-\pi / \alpha_{j}<$ $l-1-\beta_{j}<\pi / \alpha_{j}$ for $j=1, \ldots, d$.

Theorem 2.2 is a natural extension of the classical result on the $H^{l}$-regularity of the Poisson problem in smooth domains; see Lions and Magenes [18, Theorem 5.5.4], Gilbarg and Trudinger [8, Theorem 8.13], Evans [7, Theorem 6.3.5]. The following corollary is an immediate consequence of Theorem 2.2

Corollary 2.3. If $-\pi / \alpha_{j}<l-1-\beta_{j}<\pi / \alpha_{j}$ for $j=1, \ldots, d$, then the operator $\Delta: \stackrel{\circ}{V}_{2, \vec{\beta}}^{l}(\mathcal{G}) \rightarrow V_{2, \vec{\beta}}^{l-2}(\mathcal{G})$, where $l \geq 2, \vec{\beta}=\left(\beta_{1}, \ldots, \beta_{d}\right) \in \mathbb{R}^{d}$, is an isomorphism. 
3. Variational formulation of the Poisson problem in $V_{2, \vec{\beta}}^{2}(\mathcal{G})$. As noted in the introduction, in domains with smooth boundary or convex domains, the solution of the Poisson problem belongs to $H^{2}$, provided the force term is square integrable [7, 8, 18. In domains with reentrant corners, solutions suffer a loss of regularity due to singularities that develop at corners. For example, near a reentrant corner with interior angle $\alpha>\pi$, the solution behaves like $r^{\pi / \alpha}$ (where $r$ is the distance from the reentrant corner). Thus, it belongs to the Sobolev space $H^{1+\frac{\pi}{\alpha}-\varepsilon} \supset H^{2}$; see, e.g., [2, 10]. In this section we investigate variational formulations in weighted Sobolev spaces $V_{2, \vec{\beta}}^{2}$, where the application of weights at reentrant corners compensates for the solution's singularities.

We present the formulation in two equivalent variants. In the first variant, the weights are confined to isolated neighborhoods of reentrant vertices through cut-off functions that were introduced in Section 2.2. In the second variant, the cut-off functions are removed so that the weight from each corner propagates throughout the domain. The first variant is particularly appealing for computing, e.g., with finite elements, because the effects of weights remain local and do not "pollute" the bulk of the interior region. The second variant is more appealing from the analysis point of view because the clutter of the cut-off functions is removed.

Section 5] contains an application of these results to an iterative algorithm for solving the time-dependent Navier-Stokes equations.

3.1. Variational formulation with local weights. We consider the Poisson problem with homogeneous Dirichlet boundary conditions

$$
\begin{aligned}
-\Delta u=f & \text { in } \mathcal{G}, \\
u=0 & \text { on } \partial \mathcal{G},
\end{aligned}
$$

in a bounded domain $\mathcal{G} \subset \mathbb{R}^{2}$ with corners $x^{1}, \ldots, x^{d}$, as described in Section 2.2 . We pose a variational formulation for problem (3.1) in this type of domain as:

Given $f \in L_{2, \vec{\beta}}(\mathcal{G})$, find $u \in{\stackrel{\circ}{V_{2, \vec{\beta}}^{2}}}^{2}(\mathcal{G})$ such that

$$
(\Delta u, \Delta v)_{L_{2, \vec{\beta}}(\mathcal{G})}=-(f, \Delta v)_{L_{2, \vec{\beta}}(\mathcal{G})} \quad \forall v \in{\stackrel{\circ}{V_{2, \vec{\beta}}^{2}}}^{2}(\mathcal{G})
$$

TheOREM 3.1. For any $\vec{\beta}=\left(\beta_{1}, \ldots, \beta_{d}\right) \in \mathbb{R}^{d}$ such that $1-\pi / \alpha_{j}<\beta_{j}<1+\pi / \alpha_{j}$, $j=1, \ldots, d$, the variational problem (3.2) has a unique solution $u \in \stackrel{\circ}{V}_{2, \vec{\beta}}^{2}(\mathcal{G})$.

$$
\begin{aligned}
& \text { Proof. Let } \left.a: \stackrel{\circ}{V}_{2, \vec{\beta}}^{2}(\mathcal{G}) \times{\stackrel{\circ}{V_{2, \vec{\beta}}^{2}}}^{2} \mathcal{G}\right) \rightarrow \mathbb{R} \\
& \qquad a(u, v)=(\Delta u, \Delta v)_{L_{2, \vec{\beta}}(\mathcal{G})}=\int_{\mathcal{G}} \zeta_{0}^{2} \Delta u \Delta v d x+\sum_{j=1}^{d} \int_{\mathcal{K}_{j}} r_{j}^{2 \beta_{j}} \zeta_{j}^{2} \Delta u \Delta v d x,
\end{aligned}
$$

be the bilinear form corresponding to (3.2). Here $\zeta_{0}, \zeta_{1}, \ldots, \zeta_{d}$ are the cut-off functions corresponding to the corners $x^{1}, \ldots, x^{d}$, as described in Section 2.2. By applying the Cauchy-Schwarz inequality we obtain

$$
|a(u, v)| \leq\|\Delta u\|_{L_{2, \vec{\beta}}(\mathcal{G})}\|\Delta v\|_{L_{2, \vec{\beta}}(\mathcal{G})} \leq C_{1}\|u\|_{V_{2, \vec{\beta}}^{2}(\mathcal{G})}\|v\|_{V_{2, \vec{\beta}}^{2}(\mathcal{G})} \quad \forall u, v \in{\stackrel{\circ}{V_{2, \vec{\beta}}^{2}}}^{2}(\mathcal{G}),
$$


which shows the continuity of $a(\cdot, \cdot)$. From Corollary 2.3 we also have

$$
a(u, u)=\|\Delta u\|_{L_{2, \vec{\beta}}(\mathcal{G})}^{2} \geq C_{2}\|u\|_{V_{2, \vec{\beta}}^{2}(\mathcal{G})}^{2} \quad \forall u \in \dot{\circ}_{2, \vec{\beta}}^{2}(\mathcal{G}),
$$

which shows that $a(\cdot, \cdot)$ is $\stackrel{\circ}{V}_{2, \vec{\beta}}^{2}(\mathcal{G})$-coercive. In a similar way, it can be shown that the linear functional

$$
{\stackrel{\circ}{V_{2, \vec{\beta}}}}^{2}(\mathcal{G}) \ni v \mapsto-(f, \Delta v)_{L_{2, \vec{\beta}}(\mathcal{G})}=-\int_{\mathcal{G}} \zeta_{0}^{2} f \Delta v d x-\sum_{j=1}^{d} \int_{\mathcal{K}_{j}} r_{j}^{2 \beta_{j}} \zeta_{j}^{2} f \Delta v d x
$$

is bounded. The existence and uniqueness of the solution of the variational problem (3.2) follows from the Lax-Milgram lemma 7 .

3.2. Variational formulation with a global weight. In the variational formulation (3.2), the supports of the weight functions are localized near the corners through the cut-off multipliers. In this section we present an alternative variational formulation where a weight of the $r^{2 \beta}$ type extends to the entire domain. This formulation is particularly appealing when the domain has only one reentrant corner; therefore, we will limit our discussion to that case.

Thus, let us consider the Poisson problem (3.1) in a bounded domain $\mathcal{G} \subset \mathbb{R}^{2}$ with one reentrant corner and introduce the following variational formulation:

Given $f \in L_{2, \vec{\beta}}(\mathcal{G})$, find $u \in \stackrel{\circ}{V}_{2, \vec{\beta}}^{2}(\mathcal{G})$ such that

$$
\int_{\mathcal{G}} r^{2 \beta} \Delta u \Delta v d x=-\int_{\mathcal{G}} r^{2 \beta} f \Delta v d x \quad \forall v \in{\stackrel{\circ}{V_{2, \vec{\beta}}^{2}}}^{2}(\mathcal{G}),
$$

where $r$ denotes the distance from the reentrant corner and $\vec{\beta}=(\beta, 0, \ldots, 0) \in \mathbb{R}^{d}$.

LEMMA 3.2. Let $\mathcal{G}$ be a bounded domain with one reentrant corner and let $r$ be the distance from the reentrant corner. Then

$$
\|u\|_{\beta}=\left(\int_{\mathcal{G}} r^{2 \beta}|u|^{2} d x\right)^{1 / 2}
$$

is a norm equivalent to the $L_{2, \vec{\beta}}(\mathcal{G})$-norm, with $\vec{\beta}=(\beta, 0, \ldots, 0) \in \mathbb{R}^{d}$.

Proof. We will show that there exist constants $C_{1}, C_{2}>0$ such that

$$
C_{1}\|u\|_{L_{2, \vec{\beta}}(\mathcal{G})} \leq\|u\|_{\beta} \leq C_{2}\|u\|_{L_{2, \vec{\beta}}(\mathcal{G})}, \quad \forall u \in L_{2, \vec{\beta}}(\mathcal{G}) .
$$

Let $x^{1}, \ldots, x^{d}$ be the corners of $\mathcal{G}$, with $x^{1}$ the reentrant corner, and $\zeta_{j}, j=1, \ldots, d$, be the associated cut-off functions as described in Section 2.2. Recall that, with $\vec{\beta}=$ $(\beta, 0, \ldots, 0)$,

$$
\begin{aligned}
\|u\|_{L_{2, \bar{\beta}}(\mathcal{G})}^{2} & =\left\|\zeta_{0} u\right\|_{L_{2}(\mathcal{G})}^{2}+\sum_{j=1}^{d}\left\|\zeta_{j} u\right\|_{L_{2, \beta_{j}}\left(\mathcal{K}_{j}\right)}^{2} \\
& =\int_{\mathcal{G}}\left|\zeta_{0} u\right|^{2} d x+\int_{\mathcal{G} \cap \mathcal{U}_{1}} r^{2 \beta}\left|\zeta_{1} u\right|^{2} d x+\sum_{j=2}^{d} \int_{\mathcal{G} \cap \mathcal{U}_{j}}\left|\zeta_{j} u\right|^{2} d x
\end{aligned}
$$


Using $\zeta_{j}(x) \leq 1, \forall x \in \mathcal{U}_{j}, j=1, \ldots, d$, and $\zeta_{0}=0$ in some neighborhood $\mathscr{V}_{1}$ of $x^{1}$, we obtain

$$
\|u\|_{L_{2, \vec{\beta}}(\mathcal{G})}^{2} \leq c_{0} \int_{\mathcal{G} \backslash \mathscr{V}_{1}}|u|^{2} d x+c_{1} \int_{\mathcal{G} \cap U_{1}} r^{2 \beta}|u|^{2} d x+c \sum_{j=2}^{d} \int_{\mathcal{G} \cap \mathcal{U}_{j}}|u|^{2} d x .
$$

Since $r$ is bounded away from zero on $\mathcal{G} \cap \mathcal{U}_{j}, j=2, \ldots, d$, and on $\mathcal{G} \backslash \mathscr{V}_{1}$, the last estimate becomes

$$
\begin{aligned}
\|u\|_{L_{2, \vec{\beta}}(\mathcal{G})}^{2} & \leq C_{0} \int_{\mathcal{G} \backslash \mathscr{V}_{1}} r^{2 \beta}|u|^{2} d x+c_{1} \int_{\mathcal{G} \cap U_{1}} r^{2 \beta}|u|^{2} d x+\sum_{j=2}^{d} c_{j} \int_{\mathcal{G} \cap \mathcal{U}_{j}} r^{2 \beta}|u|^{2} d x \\
& \leq C\left(\int_{\mathcal{G}} r^{2 \beta}|u|^{2} d x+\int_{\mathcal{G} \cap U_{1}} r^{2 \beta}|u|^{2} d x+\sum_{j=2}^{d} \int_{\mathcal{G} \cap \mathcal{U}_{j}} r^{2 \beta}|u|^{2} d x\right) \\
& \leq C_{1}\|u\|_{\beta}^{2},
\end{aligned}
$$

which proves the first inequality in (3.5).

Next, we prove the second estimate in (3.5). We have

$$
\begin{aligned}
\|u\|_{\beta}^{2} & =\int_{\mathcal{G}} r^{2 \beta}|u|^{2} d x=\int_{\mathcal{G}} r^{2 \beta}\left(\zeta_{0}+\zeta_{1}+\cdots \zeta_{d}\right)^{2}|u|^{2} d x \\
& \leq c_{3} \int_{\mathcal{G}} r^{2 \beta}\left(\zeta_{0}^{2}+\zeta_{1}^{2}+\cdots \zeta_{d}^{2}\right)|u|^{2} d x \\
& \leq c_{4} \int_{\mathcal{G}}\left|\zeta_{0} u\right|^{2} d x+c_{3} \int_{\mathcal{G}} r^{2 \beta}\left|\zeta_{1} u\right|^{2} d x+c_{4} \sum_{j=2}^{d} \int_{\mathcal{G}}\left|\zeta_{j} u\right|^{2} d x, \\
& \leq C_{2}\|u\|_{L_{2, \vec{\beta}}(\mathcal{G})}^{2}
\end{aligned}
$$

where we used the fact that $r$ is bounded from above on $\mathcal{G}$.

TheOREm 3.3. If $1-\pi / \alpha<\beta<1+\pi / \alpha$, where $\alpha$ is the interior angle of the reentrant corner, then the variational problem (3.3) has a unique solution $u \in \stackrel{\circ}{V}_{2, \vec{\beta}}^{2}(\mathcal{G})$, with $\vec{\beta}=$ $(\beta, 0, \ldots, 0)$.

Proof. By applying Lemma 3.2 and Corollary 2.3 it can be verified that the bilinear form

$$
a: \stackrel{\circ}{V}_{2, \vec{\beta}}^{2}(\mathcal{G}) \times \stackrel{\circ}{V}_{2, \vec{\beta}}^{2}(\mathcal{G}) \rightarrow \mathbb{R}, \quad a(u, v)=\int_{\mathcal{G}} r^{2 \beta} \Delta u \Delta v d x
$$

and linear functional $\stackrel{\circ}{V}_{2, \vec{\beta}}^{2}(\mathcal{G}) \ni v \mapsto-\int_{\mathcal{G}} r^{2 \beta} f \Delta v d x$ associated with (3.3) satisfy the conditions of the Lax-Milgram lemma; thus, the assertion of the theorem follows. Details are given in 23].

3.3. Equivalence of variational formulations. The traditional $H^{1}$ variational formulation of the Poisson problem (3.1) reads:

Given $f \in H^{-1}(\mathcal{G})$, find $u \in H_{0}^{1}(\mathcal{G})$ such that

$$
\int_{\mathcal{G}} \nabla u \cdot \nabla v d x=\int_{\mathcal{G}} f v d x, \quad \forall v \in H_{0}^{1}(\mathcal{G}) .
$$


We wish to show that the unique solution of the variational problem $(\underline{3.3})$ in $\stackrel{\circ}{2}_{2, \vec{\beta}}^{2}(\mathcal{G})$, for $\beta$ in a given range, coincides with the solution of (3.6). Throughout this section, unless otherwise specified, $\overrightarrow{1}=(1,0, \ldots, 0),-\overrightarrow{1}=(-1,0, \ldots, 0), \vec{\beta}=(\beta, 0, \ldots, 0), \overrightarrow{1}-\vec{\beta}=$ $(1-\beta, 0, \ldots, 0)$. First we state and prove two auxiliary lemmas.

LEMMA 3.4. Let $\mathcal{G}$ be a bounded domain with one reentrant corner and let $r$ be the distance from the reentrant corner. Then for $\beta \leq 1$

$$
\left\{r^{-2 \beta} u: u \in H_{0}^{1}(\mathcal{G})\right\} \subset L_{2, \vec{\beta}}(\mathcal{G}) .
$$

Proof. Let $u \in H_{0}^{1}(\mathcal{G})$. In view of Lemma 3.2 , it is sufficient to show that

$$
\int_{\mathcal{G}} r^{2 \beta}\left|r^{-2 \beta} u\right|^{2} d x=\int_{\mathcal{G}} r^{-2 \beta}|u|^{2} d x<\infty .
$$

It is shown in [14, Lemma 6.6.1] that the sets $H_{0}^{1}(\mathcal{G})$ and $\left\{u \in V_{2, \overrightarrow{0}}^{1}(\mathcal{G}):\left.u\right|_{\partial \mathcal{G}}=0\right\}$ coincide. By Lemma [2.1, $V_{2, \overrightarrow{0}}^{1}(\mathcal{G})$ is continuously imbedded in $L_{2,-\vec{\beta}}(\mathcal{G})$ for $\beta \leq 1$; therefore, $\|u\|_{L_{2,-\rightarrow}(\mathcal{G})} \leq C\|u\|_{H_{0}^{1}(\mathcal{G})}$. But $\|u\|_{L_{2,-\rightarrow}(\mathcal{G})}$ is equivalent to $\int_{\mathcal{G}} r^{-2 \beta}|u|^{2} d x$ whence (3.8) follows.

The classical Green's formula $\int_{\Omega} v \Delta u d x=\int_{\partial \Omega} v \partial u / \partial n d a-\int_{\Omega} \nabla u \cdot \nabla v d x$ holds for $u \in C^{2}(\bar{\Omega}), v \in C^{1}(\bar{\Omega})$, and $\partial \Omega \in C^{1}$. Various weaker versions exist where $u$ and $v$ are moved to Sobolev spaces and the requirement of smoothness of the boundary is relaxed to some extent. See Grisvard [10] for special cases that apply to polygonal domains in $\mathbb{R}^{2}$ and Kozlov, Maz'ya, and Roßmann [14 for certain types of higher-dimension non-smooth domains. Here we prove a variant of Green's formula for domains with angular points in $\mathbb{R}^{2}$ and functions that vanish on the boundary.

Lemma 3.5. Let $\mathcal{G} \subset \mathbb{R}^{2}$ be a domain with one reentrant angle with interior angle $\alpha$. For any $\beta \in \mathbb{R}$ such that $1-\pi / \alpha<\beta<1+\pi / \alpha$, the following Green's formula holds:

$$
-\int_{\mathcal{G}} v \Delta u d x=\int_{\mathcal{G}} \nabla u \cdot \nabla v d x \quad \text { for } u \in \stackrel{\circ}{V}_{2, \vec{\beta}}^{2}(\mathcal{G}), v \in{\stackrel{\circ}{V_{2, \overrightarrow{1}}-\vec{\beta}}}^{1}
$$

Proof. We will apply a density argument. Let $\left\{u_{n}\right\}_{n=1}^{\infty}$ be a sequence in $C_{0}^{\infty}(\overline{\mathcal{G}} \backslash$ $\left.\left\{x^{1}, \ldots, x^{d}\right\}\right)$ that converges to $u$ in $\stackrel{\circ}{2}_{2, \vec{\beta}}^{2}(\mathcal{G})$, and let $\left\{v_{n}\right\}_{n=1}^{\infty}$ be a sequence in $C_{0}^{\infty}(\mathcal{G})$ that converges to $v$ in $\stackrel{\circ}{2}_{2, \overrightarrow{1}-\vec{\beta}}^{1}(\mathcal{G})$. We have

$$
-\int_{\mathcal{G}} v_{n} \Delta u_{n} d x=\int_{\mathcal{G}} \nabla u_{n} \cdot \nabla v_{n} d x \quad \text { for } n \geq 1 .
$$

Let us estimate

$$
\begin{aligned}
\mid \int_{\mathcal{G}} v_{n} \Delta u_{n} d x & -\int_{\mathcal{G}} v \Delta u d x \mid \\
& \leq \int_{\mathcal{G}}\left|v_{n} \Delta u_{n}-v_{n} \Delta u+v_{n} \Delta u-v \Delta u\right| d x \\
& \leq \int_{\mathcal{G}} r^{\beta}\left|\Delta u_{n}-\Delta u\right| r^{-\beta}\left|v_{n}\right| d x+\int_{\mathcal{G}} r^{-\beta}\left|v_{n}-v\right| r^{\beta}|\Delta u| d x \\
& \leq\left\|\Delta u_{n}-\Delta u\right\|_{\beta}\left\|v_{n}\right\|_{-\beta}+\left\|v_{n}-v\right\|_{-\beta}\|\Delta u\|_{\beta} .
\end{aligned}
$$


Since $\|\Delta u\|_{\beta}$ is equivalent to the $V_{2, \vec{\beta}}^{2}(\mathcal{G})$-norm by Corollary 2.3 and Lemma 3.2 , $\left\|\Delta u_{n}-\Delta u\right\|_{\beta} \rightarrow 0$ as $n \rightarrow \infty$. Moreover, $V_{2, \overrightarrow{1}-\vec{\beta}}^{1}(\mathcal{G})$ is continuously imbedded in $L_{2,-\vec{\beta}}$; therefore, \|\|$v_{n}-v \|_{-\beta} \rightarrow 0$ as $n \rightarrow \infty$ since $\left\{v_{n}\right\}_{n=1}^{\infty}$ converges to $v$ in $\stackrel{\circ}{2, \overrightarrow{1}-\vec{\beta}}^{1}(\mathcal{G})$. Also note that $\|\Delta u\|_{\beta}$ and $\left\|v_{n}\right\|_{-\beta}$ are bounded. Thus,

$$
\int_{\mathcal{G}} v_{n} \Delta u_{n} d x \rightarrow \int_{\mathcal{G}} v \Delta u d x \quad \text { as } n \rightarrow \infty .
$$

Next, let us estimate

$$
\begin{aligned}
\mid \int_{\mathcal{G}} \nabla u_{n} \cdot \nabla v_{n} d x & -\int_{\mathcal{G}} \nabla u \cdot \nabla v d x \mid \\
& \leq \int_{\mathcal{G}}\left|\nabla u_{n} \cdot \nabla v_{n}-\nabla u \cdot \nabla v_{n}+\nabla u \cdot \nabla v_{n}-\nabla u \cdot \nabla v\right| d x \\
& \leq\left\|\nabla u_{n}-\nabla u\right\|_{\beta-1}\left|\left\|\nabla v_{n}\right\|_{1-\beta}+\left\|\nabla v_{n}-\nabla v\right\|_{1-\beta}\right|\|\nabla u\|_{\beta-1} .
\end{aligned}
$$

Since $V_{2, \vec{\beta}}^{2}(\mathcal{G})$ is continuously imbedded in $V_{2, \vec{\beta}-\overrightarrow{1}}^{1}(\mathcal{G})$, we obtain from the estimate above

$$
\int_{\mathcal{G}} \nabla u_{n} \cdot \nabla v_{n} d x \rightarrow \int_{\mathcal{G}} \nabla u \cdot \nabla v d x \quad \text { as } n \rightarrow \infty .
$$

Finally, passing to the limit in (3.10), we obtain (3.9).

REMARK 3.6. Formula (3.9) can be extended to domains with more than one reentrant corner for $\vec{\beta}=\left(\beta_{1}, \ldots, \beta_{d}\right) \in \mathbb{R}^{d}$ such that $1-\pi / \alpha_{j}<\beta_{j}<1+\pi / \alpha_{j}, j=1, \ldots, d$, and $u \in \stackrel{\circ}{V}_{2, \vec{\beta}}^{2}(\mathcal{G}), v \in \stackrel{\circ}{V}_{2, \overrightarrow{1}-\vec{\beta}}^{1}(\mathcal{G})$, where $\overrightarrow{1}-\vec{\beta}=\left(1-\beta_{1}, \ldots, 1-\beta_{d}\right)$.

Theorem 3.7. Let $f \in L_{2, \vec{\beta}}(\mathcal{G}), 1-\pi / \alpha<\beta \leq 1$. Then the variational problem (3.3) has a unique solution in $\stackrel{\circ}{2, \vec{\beta}}^{2}(\mathcal{G})$ that coincides with the solution of the traditional variational problem (3.6).

Proof. If $f \in L_{2, \vec{\beta}}(\mathcal{G}), \beta \leq 1$, then for any $\psi \in H_{0}^{1}(\mathcal{G})$ we have

$$
\begin{aligned}
\int_{\mathcal{G}} f \psi d x & =\int_{\mathcal{G}} r^{2 \beta} f r^{-2 \beta} \psi d x=\int_{\mathcal{G}} r^{2 \beta} f \phi d x \\
& \leq\left(\int_{\mathcal{G}} r^{2 \beta} f^{2} d x\right)^{1 / 2}\left(\int_{\mathcal{G}} r^{2 \beta} \phi^{2} d x\right)^{1 / 2}<\infty
\end{aligned}
$$

where $r^{-2 \beta} \psi=\phi \in L_{2, \vec{\beta}}(\mathcal{G})$ from Lemma 3.4 Thus $f \in H^{-1}(\mathcal{G})$.

Since $\beta$ satisfies the hypothesis of Theorem 3.3. $1-\pi / \alpha<\beta<1+\pi / \alpha$, the variational problem (3.3) has a unique solution $u \in{\stackrel{\circ}{V_{2, \vec{\beta}}^{2}}}^{2}(\mathcal{G})$ that satisfies

$$
\int_{\mathcal{G}} r^{2 \beta} \Delta u \Delta v d x=-\int_{\mathcal{G}} r^{2 \beta} f \Delta v d x \quad \forall v \in \stackrel{\circ}{V}_{2, \vec{\beta}}^{2}(\mathcal{G}) .
$$

Corollary 2.3 implies

$$
\int_{\mathcal{G}} r^{2 \beta} \Delta u \phi d x=-\int_{\mathcal{G}} r^{2 \beta} f \phi d x \quad \forall \phi \in L_{2, \vec{\beta}}(\mathcal{G}) .
$$


From Lemma 3.4. $\left\{r^{-2 \beta} \psi: \psi \in H_{0}^{1}(\mathcal{G})\right\} \subset L_{2, \vec{\beta}}(\mathcal{G})$, so

or

$$
\int_{\mathcal{G}} r^{2 \beta} \Delta u r^{-2 \beta} \psi d x=-\int_{\mathcal{G}} r^{2 \beta} f r^{-2 \beta} \psi d x \quad \forall \psi \in H_{0}^{1}(\mathcal{G})
$$

$$
\int_{\mathcal{G}} \Delta u \psi d x=-\int_{\mathcal{G}} f \psi d x \quad \forall \psi \in H_{0}^{1}(\mathcal{G}) .
$$

Since $H_{0}^{1}(\mathcal{G}) \hookrightarrow \stackrel{\circ}{V}_{2, \overrightarrow{1}-\vec{\beta}}^{1}(\mathcal{G})$ for $1-\pi / \alpha<\beta \leq 1$, in view of Lemma 3.5 we may integrate by parts to obtain

$$
\int_{\mathcal{G}} \nabla u \cdot \nabla \psi d x=-\int_{\mathcal{G}} f \psi d x \quad \forall \psi \in H_{0}^{1}(\mathcal{G}),
$$

so $u$ satisfies (3.6) as asserted.

REMARK 3.8. The condition $\beta \leq 1$ in Theorem 3.7 is essential because it guarantees that the function $f \in L_{2, \vec{\beta}}(\mathcal{G})$ is in $H^{-1}(\mathcal{G})$, and hence the traditional $H^{1}$ formulation is well-posed; otherwise the statement of the theorem would make no sense.

4. Variational formulation of the Helmholtz problem in $V_{2, \vec{\beta}}^{2}(\mathcal{G})$. In this section we consider the boundary value problem

$$
\begin{aligned}
-\Delta u+\lambda u=f & & \text { in } \mathcal{G}, \\
u=0 & & \text { on } \partial \mathcal{G},
\end{aligned}
$$

where $\lambda>0$ is a constant.

4.1. Variational formulation with local weights. For a bounded domain $\mathcal{G} \subset \mathbb{R}^{2}$ with corners $x^{1}, \ldots, x^{d}$, we introduce the following variational formulation of problem (4.1):

Given $f \in L_{2, \vec{\beta}}(\mathcal{G})$, find $u \in \stackrel{\circ}{V}_{2, \vec{\beta}}^{2}(\mathcal{G})$ such that

$$
(-\Delta u+\lambda u,-\Delta v+\lambda v)_{L_{2, \vec{\beta}}(\mathcal{G})}=(f,-\Delta v+\lambda v)_{L_{2, \vec{\beta}}(\mathcal{G})} \quad \forall v \in{\stackrel{\circ}{V_{2, \vec{\beta}}^{2}}}^{2}(\mathcal{G}) .
$$

The following lemma is a well-known consequence of the Fredholm alternative for compact operators [7, 8, variants of which are used widely in the literature.

Lemma 4.1. Let $L: V \rightarrow H$ be an isomorphism, where $V, H$ are Hilbert spaces and $V$ is compactly imbedded in $H$. Then, there exists a countable set $\Lambda \subset \mathbb{R}$ such that if $\lambda \notin \Lambda$, the operator $\lambda I-L$ is an isomorphism.

Theorem 4.2. Let $\left.\mathcal{A}_{\lambda}:{\stackrel{\circ}{V_{2, \vec{\beta}}^{l}}}^{l} \mathcal{G}\right) \rightarrow V_{2, \vec{\beta}}^{l-2}(\mathcal{G}), \mathcal{A}_{\lambda}=-\Delta+\lambda I$, where $l \geq 2, \vec{\beta}=$ $\left(\beta_{1}, \ldots, \beta_{d}\right) \in \mathbb{R}^{d}$, and $-\pi / \alpha_{j}<l-1-\beta_{j}<\pi / \alpha_{j}$ for $j=1, \ldots, d$. Then, there exists a countable set $\Lambda \subset \mathbb{R}$ such that $\mathcal{A}_{\lambda}$ is an isomorphism if $\lambda \notin \Lambda$.

Proof. Since $\stackrel{\circ}{V}_{2, \beta}^{l}(\mathcal{G})$ is compactly imbedded in $V_{2, \vec{\beta}}^{l-2}(\mathcal{G})$ (Lemma 2.1) , and $\Delta: \stackrel{\circ}{V}_{2, \beta}^{l}(\mathcal{G})$ $\rightarrow V_{2, \vec{\beta}}^{l-2}(\mathcal{G})$ is an isomorphism (Corollary 2.3), the assertion follows from Lemma 4.1.

Corollary 4.3. For $\vec{\beta}=\left(\beta_{1}, \ldots, \beta_{d}\right) \in \mathbb{R}^{d}$ such that $1-\pi / \alpha_{j}<\beta_{j} \leq 1$ for $j=1, \ldots, d$, the operator $\mathcal{A}_{\lambda}: \stackrel{\circ}{V}_{2, \vec{\beta}}^{2}(\mathcal{G}) \rightarrow L_{2, \vec{\beta}}(\mathcal{G}), \mathcal{A}_{\lambda}=-\Delta+\lambda I$, is an isomorphism for any $\lambda>0$. 
Proof. Consider the problem

$$
\begin{array}{cl}
\Delta u=\lambda u & \text { in } \mathcal{G}, \\
u=0 & \text { on } \partial \mathcal{G} .
\end{array}
$$

Multiply the equation by $u$ and integrate over $\mathcal{G}$ to obtain

$$
\int_{\mathcal{G}} u \Delta u d x=\lambda \int_{\mathcal{G}} u^{2} d x
$$

Since $\beta_{j} \leq 1$ for $j=1, \ldots, d$, by Lemma $2.1 V_{2, \vec{\beta}}^{2}(\mathcal{G})$ is continuously imbedded in $V_{2, \overrightarrow{1}-\vec{\beta}}^{1}(\mathcal{G})$, where $\overrightarrow{1}-\vec{\beta}=\left(1-\beta_{1}, \ldots, 1-\beta_{d}\right)$. Therefore, according to Remark 3.6 , we can integrate by parts in (4.3) to obtain

$$
-\int_{\mathcal{G}}|\nabla u|^{2} d x=\lambda \int_{\mathcal{G}} u^{2} d x
$$

This implies that the eigenvalues of the Laplacian, as an operator $\Delta: \stackrel{\circ}{V}_{2, \vec{\beta}}^{2}(\mathcal{G}) \rightarrow L_{2, \vec{\beta}}(\mathcal{G})$, are all negative. Hence the conclusion follows from Theorem 4.2 .

Theorem 4.4. If $1-\pi / \alpha_{j}<\beta_{j}<1+\pi / \alpha_{j}$ for $j=1, \ldots, d$, then the variational problem (4.2) has a unique solution $u \in \stackrel{\circ}{2}_{2, \vec{\beta}}^{2}(\mathcal{G}), \vec{\beta}=\left(\beta_{1}, \ldots, \beta_{d}\right) \in \mathbb{R}^{d}$, for any $\lambda \notin \Lambda$.

Proof. In view of the isomorphism established in Theorem 4.2, the proof follows from the Lax-Milgram lemma, just as in Theorem 3.1 .

Corollary 4.5. If $1-\pi / \alpha<\beta_{j} \leq 1$ for $j=1, \ldots, d$, then the variational problem (4.2) has a unique solution $u \in \stackrel{\circ}{V}_{2, \vec{\beta}}^{2}(\mathcal{G}), \vec{\beta}=\left(\beta_{1}, \ldots, \beta_{d}\right) \in \mathbb{R}^{d}$, for any $\lambda>0$.

Proof. Follows immediately from Theorem 4.4 and Corollary 4.3

4.2. Variational formulation with a global weight. Our weak formulation for problem (4.1) in a bounded domain with one reentrant corner is:

Given $f \in L_{2, \vec{\beta}}(\mathcal{G})$, find $u \in \stackrel{\circ}{V}_{2, \vec{\beta}}^{2}(\mathcal{G})$ such that

$$
\int_{\mathcal{G}} r^{2 \beta}(-\Delta u+\lambda u)(-\Delta v+\lambda v) d x=\int_{\mathcal{G}} r^{2 \beta}(-\Delta v+\lambda v) f d x \quad \forall v \in \stackrel{\circ}{\dot{V}_{2, \vec{\beta}}^{2}}(\mathcal{G}),
$$

where $r$ denotes the distance from the reentrant corner and $\vec{\beta}=(\beta, 0, \ldots, 0) \in \mathbb{R}^{d}$.

Theorem 4.6. If $1-\pi / \alpha<\beta \leq 1$, where $\alpha$ is the interior angle of the reentrant corner, then the variational problem (4.4) has a unique solution $u \in{\stackrel{\circ}{V_{2, \vec{\beta}}^{2}}}^{2}(\mathcal{G})$, with $\vec{\beta}=$ $(\beta, 0, \ldots, 0)$.

Proof. As before, we apply the Lax-Milgram lemma. The bilinear form corresponding to (4.4) is defined as

$$
a: \stackrel{\circ}{V}_{2, \vec{\beta}}^{2}(\mathcal{G}) \times \stackrel{\circ}{V}_{2, \vec{\beta}}^{2}(\mathcal{G}) \rightarrow \mathbb{R}, \quad a(u, v)=\int_{\mathcal{G}} r^{2 \beta}(-\Delta u+\lambda u)(-\Delta v+\lambda v) d x .
$$


The bilinear form is continuous because for any $u, v \in{\stackrel{\circ}{V_{2, \vec{\beta}}^{2}}}^{2}(\mathcal{G})$ we have

$$
\begin{aligned}
|a(u, v)| & \leq\|-\Delta u+\lambda u\|_{\beta}\|-\Delta v+\lambda v\|_{\beta} \\
& \leq c\|-\Delta u+\lambda u\|_{L_{2, \beta}(\mathcal{G})}\|-\Delta v+\lambda v\|_{L_{2, \beta}(\mathcal{G})} \leq C\|u\|_{V_{2, \beta}^{2}(\mathcal{G})}\|v\|_{V_{2, \beta}^{2}(\mathcal{G})},
\end{aligned}
$$

where we used Lemma 3.2 and Corollary 4.3, To prove the coercivity of the bilinear form, we estimate

$$
a(u, u)=\|-\Delta u+\lambda u\|_{\beta}^{2} \geq C_{1}\|-\Delta u+\lambda u\|_{L_{2, \vec{\beta}}(\mathcal{G})}^{2} \geq C_{2}\|u\|_{V_{2, \vec{\beta}}^{2}(\mathcal{G})}^{2} \forall u \in{\stackrel{\circ}{V_{2, \vec{\beta}}}}^{2}(\mathcal{G}),
$$

where again we used Lemma 3.2 and Corollary 4.3. Since the linear functional $v \mapsto$ $\int_{\mathcal{G}} r^{2 \beta}(-\Delta v+\lambda v) f d x$ is bounded on $\stackrel{\circ}{V}_{2, \vec{\beta}}^{2}(\mathcal{G})$, the existence and uniqueness of solution to (4.4) follow.

4.3. Equivalence of variational formulations. The traditional $H^{1}$ formulation of problem (4.1) reads:

Given $f \in H^{-1}(\mathcal{G})$, find $u \in H_{0}^{1}(\mathcal{G})$ such that

$$
\int_{\mathcal{G}} \nabla u \cdot \nabla v d x+\lambda \int_{\mathcal{G}} u v d x=\int_{\mathcal{G}} f v \quad \forall v \in H_{0}^{1}(\mathcal{G}) .
$$

Theorem 4.7. Let $f \in L_{2, \vec{\beta}}(\mathcal{G}), 1-\pi / \alpha<\beta \leq 1$, and $\lambda>0$. Then the unique solution of the variational problem (4.4) in $\stackrel{\circ}{V}_{2, \vec{\beta}}^{2}(\mathcal{G})$ coincides with the solution of the variational problem (4.6).

Proof. Let $u \in \stackrel{\circ}{V}_{2, \vec{\beta}}^{2}(\mathcal{G})$ be the unique solution of the variational problem (4.4). Then, $u$ satisfies

$$
\int_{\mathcal{G}} r^{2 \beta}(-\Delta u+\lambda u)(-\Delta v+\lambda v) d x=\int_{\mathcal{G}} r^{2 \beta}(-\Delta v+\lambda v) f d x \quad \forall v \in \stackrel{\circ}{V}_{2, \vec{\beta}}^{2}(\mathcal{G}) .
$$

Corollary 4.3 implies

$$
\int_{\mathcal{G}} r^{2 \beta}(-\Delta u+\lambda u) \phi d x=\int_{\mathcal{G}} r^{2 \beta} f \phi d x \quad \forall \phi \in L_{2, \vec{\beta}}(\mathcal{G}) .
$$

From Lemma 3.4. $\left\{r^{-2 \beta} \psi: \psi \in H_{0}^{1}(\mathcal{G})\right\} \subset L_{2, \vec{\beta}}(\mathcal{G})$, so

$$
\int_{\mathcal{G}} r^{2 \beta}(-\Delta u+\lambda u) r^{-2 \beta} \psi d x=\int_{\mathcal{G}} r^{2 \beta} f r^{-2 \beta} \psi d x \quad \forall \psi \in H_{0}^{1}(\mathcal{G})
$$

that is

$$
\int_{\mathcal{G}}(-\Delta u+\lambda u) \psi d x=\int_{\mathcal{G}} f \psi d x \quad \forall \psi \in H_{0}^{1}(\mathcal{G}) .
$$

By Lemma 3.5 since $H_{0}^{1}(\mathcal{G}) \hookrightarrow V_{2, \overrightarrow{1}-\vec{\beta}}^{1}(\mathcal{G})$ for $1-\pi / \alpha<\beta \leq 1$, we can integrate by parts to obtain

$$
\int_{\mathcal{G}} \nabla u \cdot \nabla \psi d x+\lambda \int_{\mathcal{G}} u \psi d x=\int_{\mathcal{G}} f \psi d x \quad \forall \psi \in H_{0}^{1}(\mathcal{G}),
$$

which shows that $u$ is the solution of the problem (4.6). 
5. Numerical scheme for the Navier-Stokes equations in weighted Sobolev spaces. We consider the Navier-Stokes equations in a bounded domain $\mathcal{G} \subset \mathbb{R}^{2}$, with no-slip boundary conditions

$$
\begin{aligned}
\mathbf{u}_{t}+(\nabla \mathbf{u}) \mathbf{u}+\nabla p=\nu \Delta \mathbf{u}+\mathbf{f} & \text { in } \mathcal{G} \times(0, T] \\
\operatorname{div} \mathbf{u}=0 & \text { in } \mathcal{G} \times(0, T] \\
\mathbf{u}=\mathbf{0} & \text { on } \partial \mathcal{G} \times(0, T], \\
\mathbf{u}=\mathbf{u}_{0} & \text { at } t=0
\end{aligned}
$$

where $\mathbf{u}$ is the fluid velocity, $p$ is the pressure, $\nu$ is the kinematic viscosity, and $\mathbf{f}$ is an external force.

In [19], Liu, Liu, and Pego have proposed a numerical scheme based on a timediscretization implicit in viscosity and explicit in pressure and convection terms that works as follows:

Given an approximation $\mathbf{u}^{n} \in\left[H^{2}(\mathcal{G}) \cap H_{0}^{1}(\mathcal{G})\right]^{2}$ to the velocity at the $n^{\text {th }}$ time step, determine $p^{n} \in H^{1}(\mathcal{G})$ from a weak-form Poisson equation

$$
\left\langle\nabla p^{n}, \nabla \phi\right\rangle=\left\langle\mathbf{f}^{n}-\left(\nabla \mathbf{u}^{n}\right) \mathbf{u}^{n}+\nu \Delta \mathbf{u}^{n}-\nu \nabla \operatorname{div} \mathbf{u}^{n}, \nabla \phi\right\rangle \quad \forall \phi \in H^{1}(\mathcal{G}),
$$

then determine $\mathbf{u}^{n+1} \in\left[H^{2}(\mathcal{G}) \cap H_{0}^{1}(\mathcal{G})\right]^{2}$ from the Helmholtz problem

$$
\begin{array}{r}
\frac{\mathbf{u}^{n+1}-\mathbf{u}^{n}}{k}-\nu \Delta \mathbf{u}^{n+1}=\mathbf{f}^{n}-\left(\nabla \mathbf{u}^{n}\right) \mathbf{u}^{n}-\nabla p^{n} \\
\left.\mathbf{u}^{n+1}\right|_{\partial \mathcal{G}}=\mathbf{0} .
\end{array}
$$

Here $\langle\cdot, \cdot\rangle$ denotes the inner product in $L_{2}$ and $k$ is the time step. The weak form of the latter equation is:

$$
\begin{array}{r}
\left\langle-\Delta \mathbf{u}^{n+1}+\frac{1}{\nu k} \mathbf{u}^{n+1}, \Delta \boldsymbol{\Psi}\right\rangle=\left\langle\frac{1}{\nu k} \mathbf{u}^{n}+\frac{1}{\nu}\left(\mathbf{f}^{n}-\left(\nabla \mathbf{u}^{n}\right) \mathbf{u}^{n}+\nabla p^{n}\right), \Delta \boldsymbol{\Psi}\right\rangle, \\
\forall \boldsymbol{\Psi} \in\left[H^{2}(\mathcal{G}) \cap H_{0}^{1}(\mathcal{G})\right]^{2} .
\end{array}
$$

It is shown in [19] that if the boundary is $C^{3}$, then the velocity iterates converge in $H^{2}$. Moreover, the corresponding fully discrete finite element scheme, with $C^{1}$ elements for velocity and $C^{0}$ elements for pressure, is unconditionally stable and does not require any compatibility conditions between the finite element spaces for the velocity and pressure.

The condition on the smoothness of the boundary may be relaxed somewhat. Kellogg and Osborn [1] have shown that in convex polygonal domains (see Dauge [5] for convex polyhedra) the velocity field corresponding to the Navier-Stokes equations is in $H^{2}$. We have applied the numerical scheme (5.2)-(5.3) to several problems on convex polygons and obtained the expected solutions for moderate Reynolds numbers. In non-convex polygons, however, the velocity develops singularities at reentrant corners and no longer is in $H^{2}$; therefore, equation (5.4) ceases to make sense. For domains of this type we reformulate the scheme (5.2)-(5.3) in weighted Sobolev spaces, along the ideas described in Section 4. Specifically, we propose: 
Given an approximation $\mathbf{u}^{n} \in\left[{\stackrel{\circ}{V_{2, \vec{\beta}}^{2}}}^{2}(\mathcal{G})\right]^{2}$ to the velocity at the $n^{\text {th }}$ time step, determine $p^{n} \in V_{2, \vec{\beta}}^{1}(\mathcal{G})$ from

$$
\left(\nabla p^{n}, \nabla \phi\right)_{L_{2, \vec{\beta}}(\mathcal{G})}=\left(\mathbf{f}^{n}-\left(\nabla \mathbf{u}^{n}\right) \mathbf{u}^{n}+\nu \Delta \mathbf{u}^{n}-\nu \nabla \operatorname{div} \mathbf{u}^{n}, \nabla \phi\right)_{L_{2, \vec{\beta}}(\mathcal{G})} \forall \phi \in V_{2, \vec{\beta}}^{1}(\mathcal{G}),
$$

then determine $\left.\mathbf{u}^{n+1} \in\left[{\stackrel{\circ}{V_{2, \vec{\beta}}^{2}}}^{2} \mathcal{G}\right)\right]^{2}$ from

$$
\begin{aligned}
\left(-\Delta \mathbf{u}^{n+1}+\lambda \mathbf{u}^{n+1},-\Delta \Psi+\lambda \boldsymbol{\Psi}\right)_{L_{2, \vec{\beta}}(\mathcal{G})} & \\
=\left(\lambda \mathbf{u}^{n}+\lambda k\left(\mathbf{f}^{n}-\left(\nabla \mathbf{u}^{n}\right) \mathbf{u}^{n}-\nabla p^{n}\right),-\Delta \Psi+\lambda \Psi\right)_{L_{2, \vec{\beta}}(\mathcal{G})} & \forall \boldsymbol{\Psi} \in\left[\stackrel{\circ}{V}_{2, \vec{\beta}}^{2}(\mathcal{G})\right]^{2},
\end{aligned}
$$

where $\lambda=1 / \nu k$.

Osborn 21] has shown that the singularity of the velocity field near a reentrant corner with interior angle $\alpha$ is related to the roots of the equations $\sinh ^{2} z \alpha-z^{2} \sin ^{2} \alpha=0$ in the complex plane. In particular, when $\alpha=3 \pi / 2$, the velocity is $O\left(r^{\mu}\right)$, where $\mu \approx 0.5444837368$. In the numerical simulations presented in Section 6.3 we use $\vec{\beta}=$ $(1,0, \ldots, 0)$, which falls within the range stipulated in Theorem 4.6,

The well-posedness of the variational problems in (5.5) -(5.6) follows from the results in Sections 3 and 4. No proof of convergence of the iterative scheme (5.5)-(5.6) is available at this time but numerical experiments (see Section 6) indicate that the proposed scheme produces the expected solution.

6. Numerical experiments. We have made extensive numerical studies of the problems in Sections 3 5. using a software that we have developed for this purpose. The software, documented in a forthcoming book [22, implements a $C^{1}$-compatible finite element scheme over an unstructured triangular mesh in arbitrary polygonal domains. The basis elements consist of fifth-degree Argyris polynomials [3. In the following subsections we will describe numerical results obtained for several test problems.

6.1. The Poisson problem in an L-shaped domain. Consider the problem

$$
\begin{aligned}
-\Delta u=f & \text { in } \mathcal{G}, \\
u=0 & \text { on } \partial \mathcal{G},
\end{aligned}
$$

in the L-shaped domain $\mathcal{G}=(-1,1)^{2} \backslash[0,1] \times[-1,0]$. We choose

$$
f=\frac{4}{3} r^{2 / 3}\left[\left(10-4 r^{2}\right) \sin \frac{2}{3} \theta-r^{2} \sin \frac{10}{3} \theta\right]
$$

which corresponds to the exact solution

$$
u=2\left(1-x_{1}^{2}\right)\left(1-x_{2}^{2}\right) r^{2 / 3} \sin \frac{2}{3} \theta
$$

where $r, \theta$ are the polar coordinates associated with the Cartesian coordinates $x_{1}, x_{2}$. Here and in the sequel $A$ denotes the area of the largest element in an unstructured 
triangular mesh, $h=\sqrt{A}$, and $u_{h}$ denotes the corresponding finite element solution. We let $\overrightarrow{1}=(1,0, \ldots, 0) \in \mathbb{R}^{6}$ and recall the notations:

$$
\begin{gathered}
\left\|u-u_{h}\right\|_{L_{2, \overrightarrow{1}}}=\left(\int_{\mathcal{G}} r^{2}\left|u-u_{h}\right|^{2} d x\right)^{1 / 2} \\
\left\|u-u_{h}\right\|_{V_{2, \overrightarrow{1}}^{2}}=\left(\int_{\mathcal{G}}\left(r^{2}\left|\nabla \nabla u-\nabla \nabla u_{h}\right|^{2}+\left|\nabla u-\nabla u_{h}\right|^{2}+r^{-2}\left|u-u_{h}\right|^{2}\right) d x\right)^{1 / 2}, \\
\left\|u-u_{h}\right\|_{a, \overrightarrow{1}}=\left(\int_{\mathcal{G}} r^{2}\left|\Delta u-\Delta u_{h}\right|^{2} d x\right)^{1 / 2} .
\end{gathered}
$$

Figure 1 shows the sequence of uniform meshes used in computations. In Figure 2 we

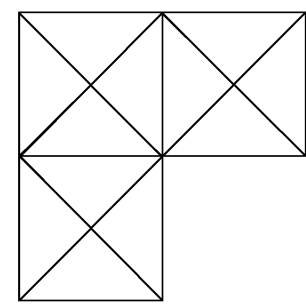

(a) $A=0.4$

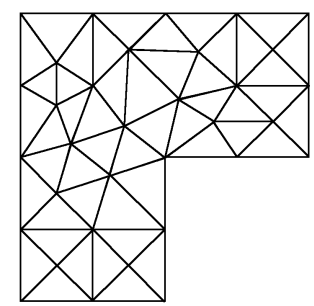

(b) $A=0.1$

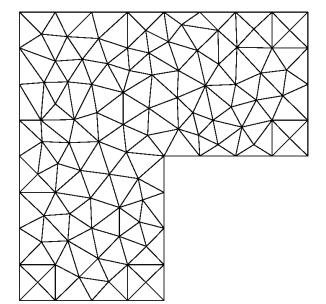

(c) $A=0.025$

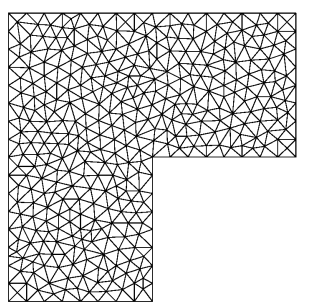

(d) $A=0.00625$

Fig. 1. Four unstructured uniform triangulations of the L-shaped domain $\mathcal{G}=(-1,1)^{2} \backslash[0,1] \times[-1,0]$. In each case, $A$ is the area of the largest element.

plot the error in various norms as functions of $h$ in a loglog plot. The convergence of the finite element approximations is proved in 23. Optimal rates of convergence on graded (non-uniform) meshes are derived in [24].

It can be seen in Figure 2 that the experimental rate of convergence in the $V_{2, \overrightarrow{1}}^{2}$ norm is approximately $2 / 3$. The same rate of convergence is observed for the $H^{1}$ norm. This result is consistent with the rate of convergence expected for the traditional $H^{1}$ formulation, when uniform meshes are used. In 24] we derive error estimates in the $V_{2, \vec{\beta}}^{2}$ norm and design graded meshes that yield optimal convergence rates.

6.2. Helmholtz equation in an L-shaped domain. We consider the boundary value problem

$$
\begin{aligned}
-\Delta u+\lambda u=f & \text { in } \mathcal{G}, \\
u=0 & \text { on } \partial \mathcal{G},
\end{aligned}
$$

with $\lambda>0$ constant, in the L-shaped domain $\mathcal{G}=(-1,1)^{2} \backslash[0,1] \times[-1,0]$. We choose $f$ such that the exact solution of the problem is given by (6.2).

We have performed numerical studies for various values of the parameter $\lambda$. We summarize here the results obtained for $\lambda=3$, noting that similar results were obtained for other values of $\lambda$. For this problem $\|u\|_{a, \overrightarrow{1}}=\left(\int_{\mathcal{G}} r^{2}|\Delta u-\lambda u|^{2} d x\right)^{1 / 2}$. The meshes used in our computations are the same as in the previous section and are plotted in Figure 1 Figure 3 shows the errors as functions of $h$ in a loglog plot. The experimental convergence rates observed are similarly obtained for the Poisson problem. 


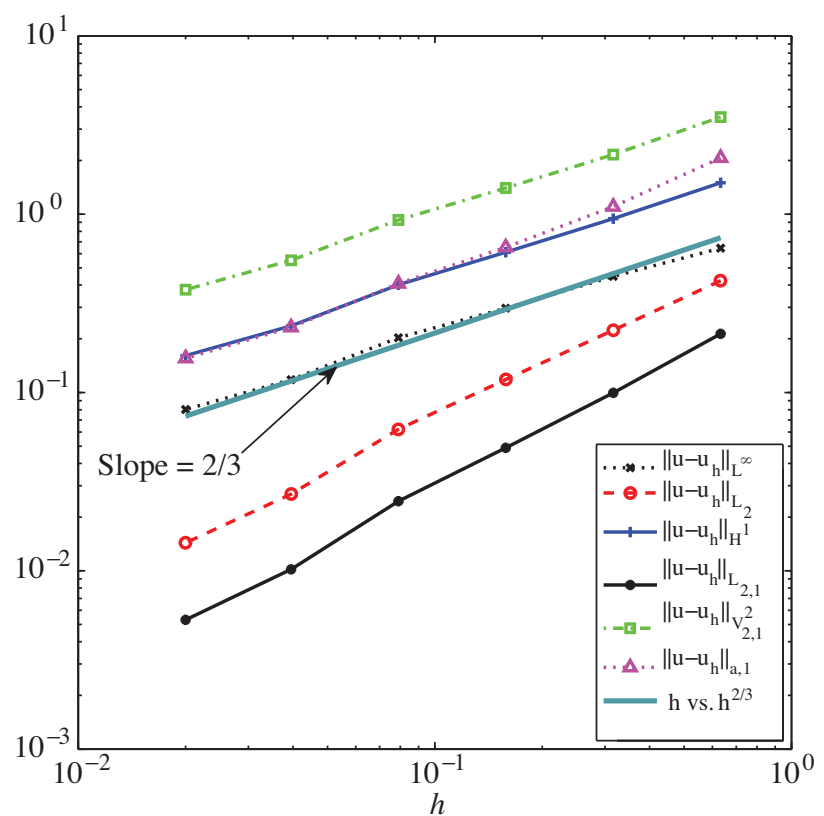

FIG. 2. Log-log plot of the error for the Poisson problem in various norms giving experimental rates of convergence as respective slopes. A line with slope $2 / 3$ is displayed for comparison.

6.3. Navier-Stokes equations: Backstep flow. In this section we solve a classical benchmark problem in fluid dynamics, the backstep problem, using the scheme described in (5.5) - (5.6) .

The diagram in Figure 4 depicts a flow region where the fluid enters from the left, flows over a step, and leaves to the right. We prescribe parabolic velocity profiles at the inlet and outlet, and zero velocities on the rest of the boundary. Specifically, in the simulations presented here, we use $\mathbf{f}=\mathbf{0}, \nu=1$, and

$$
\begin{array}{rll}
u_{1}=128 x_{2}\left(1-x_{2}\right), & u_{2}=0 & \text { at } x_{1}=-1, \\
u_{1}=16\left(1+x_{2}\right)\left(1-x_{2}\right), & u_{2}=0 & \text { at } x_{1}=3 .
\end{array}
$$

This corresponds to a Reynolds number $R e \approx 43$, where $R e=2 H U_{\text {avg }} / \nu$. Here $H=1$ is the height of the step and $U_{\text {avg }}$ is the average velocity at the inlet.

No exact solution is available for flow over a backstep; therefore, we compare our solution against that produced by the commercial software package Comsol. In Figures 5 (a), (c), (e), and (g), we show the graphs of the $x$ and $y$ components of the velocity, pressure, and divergence obtained with our weighted $H^{2}$ solver, and in Figures 5 (b), (d), (f), and (h), we show the corresponding graphs produced by ComsoL's $H^{1}$ solver. These results were obtained by solving the time-dependent Navier-Stokes equations until steady-state was reached. 


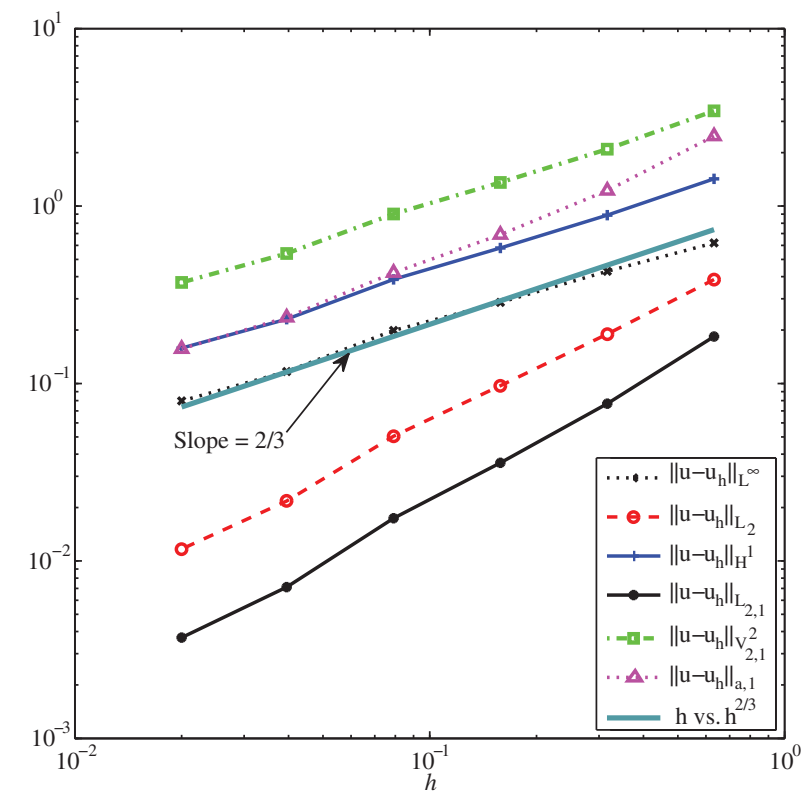

FIG. 3. Log-log plot of the error in various norms for the Helmholtz problem, $\lambda=3$, giving experimental rates of convergence as respective slopes. A line with slope $2 / 3$ is displayed for comparison.

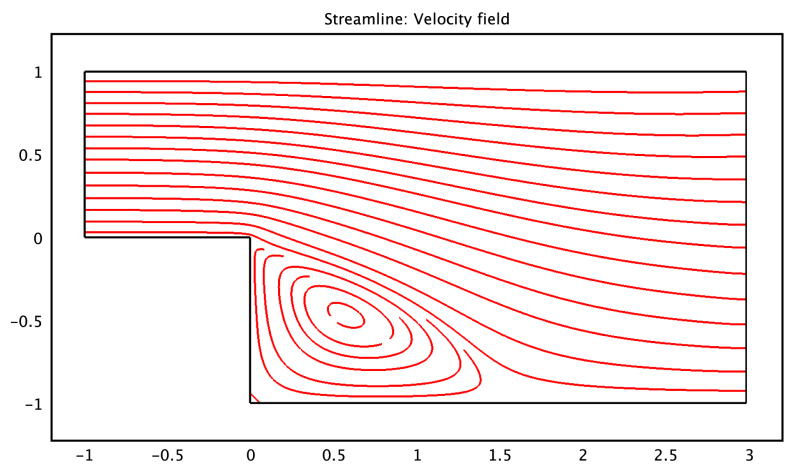

FIG. 4. Streamlines in the backstep flow.

We view the numerical results presented in this section as experiments illustrating the viability of our variational formulations, rather than a proof of superiority of our approach to those of others.

7. Acknowledgments. This article is partially based on the doctoral dissertation 23. of the first author at the University of Maryland, Baltimore County. The author gratefully acknowledges the financial support from the University of Maryland, Baltimore County during the course of her studies. 


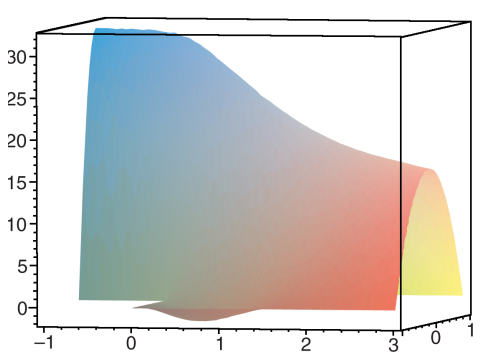

(a) $x$-velocity: Our solver

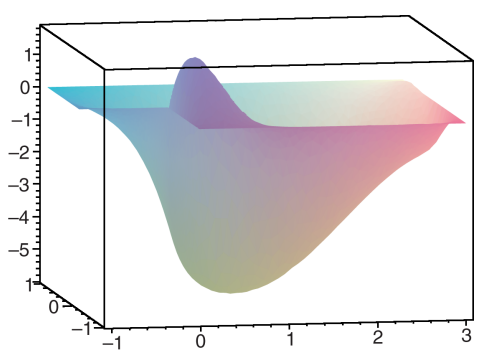

(c) $y$-velocity: Our solver

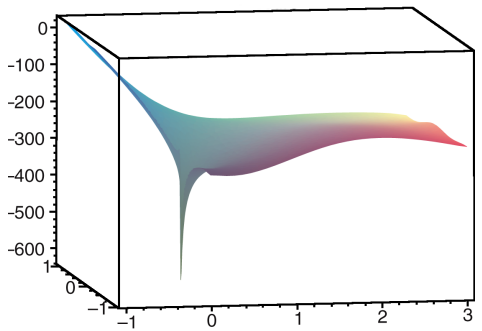

(e) Pressure: Our solver

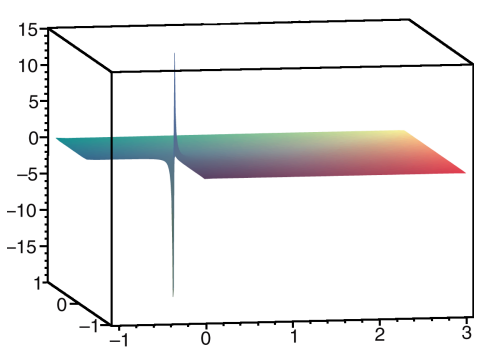

(g) Divergence: Our solver

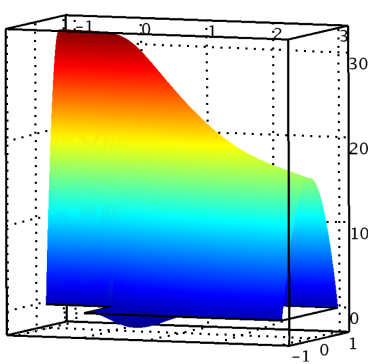

(b) $x$-velocity: COMSOL

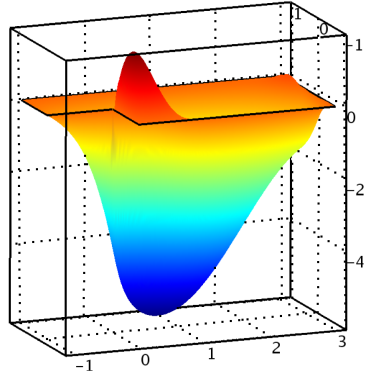

(d) $y$-velocity: COMSOL

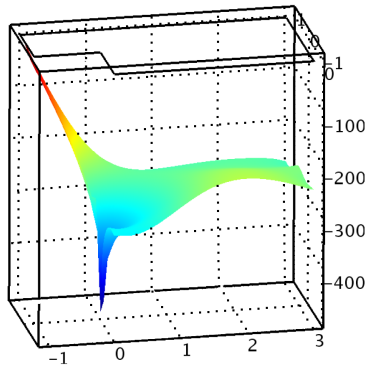

(f) Pressure: COMSOL

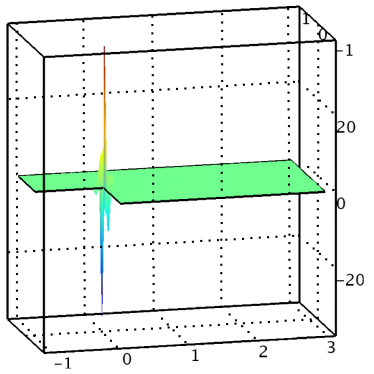

(h) Divergence: COMSOL

FIG. 5. Backstep flow: Steady-state results.

\section{REFERENCES}

[1] A. K. Aziz and R. B. Kellogg, On homeomorphisms for an elliptic equation in domains with corners, Differential Integral Equations 8 (1995), no. 2, 333-352. MR1296128 (95j:35058) 
[2] Graham F. Carey and J. Tinsley Oden, Finite elements. Vol. II, The Texas Finite Element Series, II, Prentice Hall Inc., Englewood Cliffs, NJ, 1983, A second course. MR767804 (86m:65001b)

[3] Philippe G. Ciarlet, The finite element method for elliptic problems, Classics in Applied Mathematics, vol. 40, Society for Industrial and Applied Mathematics (SIAM), Philadelphia, PA, 2002, Reprint of the 1978 original (North-Holland, Amsterdam); MR 0520174 (58:25001)

[4] Monique Dauge, Elliptic boundary value problems on corner domains, Lecture Notes in Mathematics, vol. 1341, Springer-Verlag, Berlin, 1988, Smoothness and asymptotics of solutions. MR961439 (91a:35078)

[5] , Stationary Stokes and Navier-Stokes systems on two- or three-dimensional domains with corners. I. Linearized equations, SIAM J. Math. Anal. 20 (1989), no. 1, 74-97. MR977489 (90b:35191)

[6] - Singularities of corner problems and problems of corner singularities, Actes du 30ème Congrès d'Analyse Numérique: CANum '98 (Arles, 1998), ESAIM Proc., vol. 6, Soc. Math. Appl. Indust., Paris, 1999, pp. 19-40 (electronic). MR.1689440 (2001g:35054)

[7] Lawrence C. Evans, Partial differential equations, Graduate Studies in Mathematics, vol. 19, American Mathematical Society, Providence, RI, 1998. MR.1625845 (99e:35001)

[8] David Gilbarg and Neil S. Trudinger, Elliptic partial differential equations of second order, second ed., Grundlehren der Mathematischen Wissenschaften [Fundamental Principles of Mathematical Sciences], vol. 224, Springer-Verlag, Berlin, 1983. MR737190 (86c:35035)

[9] P. Grisvard, Elliptic problems in nonsmooth domains, Monographs and Studies in Mathematics, vol. 24, Pitman (Advanced Publishing Program), Boston, MA, 1985. MR775683 (86m:35044)

[10] _ Singularities in boundary value problems, Recherches en Mathématiques Appliquées [Research in Applied Mathematics], vol. 22, Masson, Paris, 1992. MR.1173209 (93h:35004)

[11] R. B. Kellogg and J. E. Osborn, A regularity result for the Stokes problem in a convex polygon, J. Functional Analysis 21 (1976), no. 4, 397-431. MR0404849 (53:8649)

[12] V. A. Kondrat'ev, Boundary value problems for elliptic equations in domains with conical or angular points, Transactions of the Moscow Mathematical Society for the year 1967, volume 16, American Mathematical Society, Providence, R.I., 1968, pp. 227-313. MR0226187 (37:1777)

[13] V. A. Kondrat'ev and O. A. Olĕnik, Boundary value problems for partial differential equations in nonsmooth domains, Russian Mathematical Surveys 38 (1983), 1-86. MR695471 (85j:35002)

[14] V. A. Kozlov, V. G. Maz'ya, and J. Rossmann, Elliptic boundary value problems in domains with point singularities, Mathematical Surveys and Monographs, vol. 52, American Mathematical Society, Providence, R.I., 1997. MR.1469972 (98f:35038)

[15] _ Spectral problems associated with corner singularities of solutions to elliptic equations, Mathematical Surveys and Monographs, vol. 85, American Mathematical Society, Providence, R.I., 2001. MR 1788991 (2001i:35069)

[16] Alois Kufner, Weighted Sobolev spaces, BSB B. G. Teubner Verlagsgesellschaft, Leipzig, 1980. MR664599 (84e:46029)

[17] Alois Kufner and Anna-Margarete Sändig, Some applications of weighted Sobolev spaces, TeubnerTexte zur Mathematik [Teubner Texts in Mathematics], vol. 100, BSB B. G. Teubner Verlagsgesellschaft, Leipzig, 1987, With German, French and Russian summaries. MR926688 (89h:35096)

[18] J.-L. Lions and E. Magenes, Non-homogeneous boundary value problems and applications. Vol. I, Springer-Verlag, New York, 1972, Translated from the French by P. Kenneth, Die Grundlehren der mathematischen Wissenschaften, Band 181.

[19] Jian-Guo Liu, Jie Liu, and Robert L. Pego, Stability and convergence of efficient Navier-Stokes solvers via a commutator estimate, Comm. Pure Appl. Math. 60 (2007), no. 10, 1443-1487. MR 2342954 (2008k:76039)

[20] Serge Nicaise, Regularity of the solutions of elliptic systems in polyhedral domains, Bull. Belg. Math. Soc. Simon Stevin 4 (1997), no. 3, 411-429. MR1457079 (98k:35044)

[21] John E. Osborn, Regularity of solutions of the Stokes problem in a polygonal domain, Numerical solution of partial differential equations, III (Proc. Third Sympos. (SYNSPADE), Univ. Maryland, College Park, Md., 1975), Academic Press, New York, 1976, pp. 393-411. MR0467032 (57:6901)

[22] Rouben Rostamian and Ana Maria Soane, Incompressible Fluid Flow and $C^{1}$ Finite Elements, Book, in preparation, 2009.

[23] Ana Maria Soane, Variational problems in weighted Sobolev spaces with applications to Computational Fluid Dynamics, Ph.D. thesis, University of Maryland, Baltimore County, 2008. 
[24] Ana Maria Soane, Manul Suri, and Rouben Rostamian, The optimal convergence rate of a $C^{1}$ finite element method for non-smooth domains, Journal of Computational and Applied Mathematics 233 (2010), no. 10, 2711-2723.

[25] T. von Petersdorff and E. P. Stephan, Decompositions in edge and corner singularities for the solution of the Dirichlet problem of the Laplacian in a polyhedron, Math. Nachr. 149 (1990), 71103. MR:1124795 (92h:35053) 